\title{
A STUDY OF SCAN PATTERNS FOR MOBILE MAPPING
}

\author{
Jan Elseberg ${ }^{\mathrm{a}}$, Dorit Borrmann ${ }^{\mathrm{b}}$ and Andreas Nüchter ${ }^{\mathrm{b} *}$ \\ ${ }^{a}$ School of Engineering and Science, Automation Group, Jacobs University of Bremen gGmbH, \\ Campus Ring 1, Bremen 28759, Germany \\ b Informatics VII : Robotics and Telematics, Julius-Maximilians-University Würzburg, \\ Am Hubland, Wüzburg 97074, Germany \\ andreas@nuechti.de, http://www.nuechti.de
}

KEY WORDS: LIDAR, scan patterns, mobile mapping, combination of terrestrial and kinemantic scanning

\begin{abstract}
:
Mobile terrestrial scanning systems automate terrestrial laser scanning. Continous scanning mobile terrestrial systems constantly spin the terrestrial laser scanner and thus combine terrestrial scanning with kinematic laser scanning. This paper presents a scan pattern analysis for these systems. We aim at finding the most advantageous combination of terrestrial and kinematic systems. The resulting $3 \mathrm{D}$ point cloud depends on the scan pattern and the trajectory and velocity of the mobile system.
\end{abstract}

\section{INTRODUCTION}

Motivated by the increasing need of rapid characterization of environments in 3D, we designed a robot system that automates the work of an operator of terrestrial laser scanners. The constructed system allows working without the use of targets or markers and thus enables the surveyors to save more than $75 \%$ of the time spent in the field. Another impulse for developing the platform is the demand for a remote inspection tool. The robot is able to survey remote sites or danger areas, such as plants, underground mines, tunnels and caves, or channels in a stop-scan-and-go fashion. The availability of the robotic platform further enables us to study mobile laser scan systems. Making the step from mobile terrestrial scanning systems to continuous scanning mobile terrestrial systems, yields another level of automation. These systems constantly spin the terrestrial laser scanner and thus combine the advantages of terrestrial scanning with those of kinematic laser scanning. With the first systems ready for being employed in the field, we analyze their scanning patterns. It turns out that this has a major impact on the resulting $3 \mathrm{D}$ point cloud.

This paper presents the recently developed robot Irma3D cf. Fig. 1. From a detailed analysis of scan patterns, we state advantages and disadvantages and formulate a scanning method for a backpack based 3D scanning system, cf. Fig 1 right. We briefly summarize our mobile mapping solutions with constantly spinning $3 \mathrm{D}$ scanners and present results.

\section{MOBILE MAPPING SYSTEMS}

\subsection{The robot Irma3D}

The Intelligent Robot for Mapping Applications in 3D (Irma3D) is a robotic mobile laser scanner that was developed for the purpose of exploring issues like registration and calibration in a mobile laser scanning scenario. Irma3D is a small, battery-powered, light weight, three wheeled vehicle. Irma3D and its components are depicted in Fig. 2. With a width of $52 \mathrm{~cm}$ it is small enough to pass through narrow doorways. The three-wheeled design allows for a high maneuverability such that it can rotate on the spot. These properties make Irma3D ideally suited to indoor environments. In addition, the high-powered electrical two-wheel drive powered by two $150 \mathrm{~W}$ DC motors by Maxon with a top speed of

${ }^{*}$ Corresponding author. about $2.2 \mathrm{~m} / \mathrm{s}$ combined with the $26 \mathrm{~cm}$ wide pneumatic wheels also make it capable of operating in moderately challenging outdoor environments. The robot can be remote-controlled, either via a W-LAN connection or through a gamepad or similar devices. Irma3D can also be used in a fully autonomous mode. Once activated, Irma3D will attempt to explore its surroundings, up to some preset limits, and create a 3D map of the environment.

As a laser scanner platform, it can be used to acquire range measurements while moving through the environment. Alternatively, the robot can remain stationary when a $3 \mathrm{D}$ point cloud is acquired. This type of static laser scanning is called stop-and-go scanning. It is possible to create $3 \mathrm{D}$ models of the environment as detailed as with mobile laser scanning. However, since the laser scanner is not operating while the robot is moving, more time is required in this mode to create equally large point clouds. This dual-use of Irma3D is made possible by the 3D terrestrial laser range finder that it is equipped with. Without a 3D scanner that is able to freely rotate, Irma3D could not acquire 3D range images of its environment without moving.

The robot Irma3D is a combination of several sensors, a mobile platform and a portable laptop for processing data and controlling the robot itself. The chassis of the robot is a modified Volksbot RT 3. The Volksbot RT 3 has two front wheels. Each is actuated by a single Maxon servo motor. Together, the two motors are powerful enough to move the robot at a total maximum velocity of $2.2 \mathrm{~m} / \mathrm{s}$. The third wheel is in the back of the chassis and is swivel-mounted and thus completely passive as it follows the directions of the front wheels. The platform is powered by four

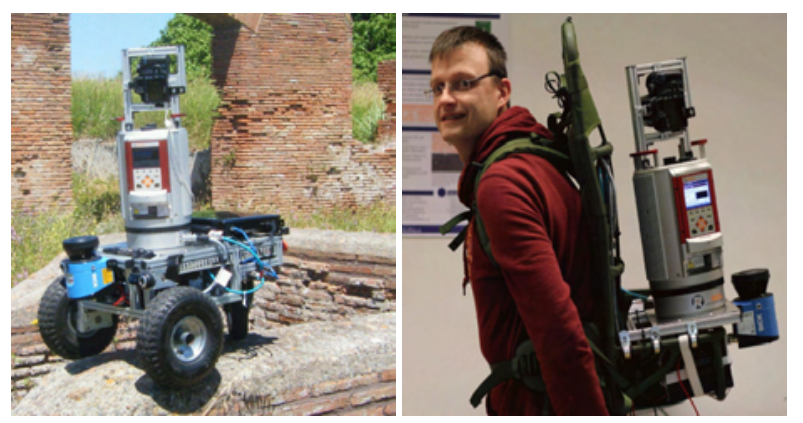

Figure 1: Left: The robot Irma3D. Videos of the system are available at http://www.youtube.com/AutomationAtJacobs Right: The backpack mobile mapping system. 


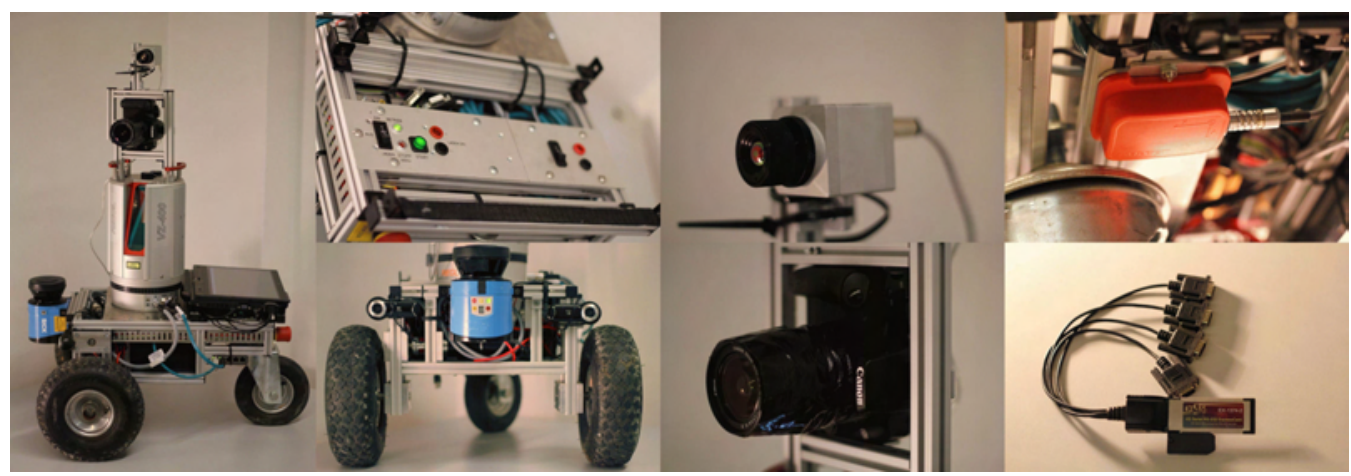

Figure 2: Images of Irma3D. Left: Side view of Irma3D with all of its sensors and equipment. Top left: The control panel with switches for the two electric circuits, sockets for loading the batteries and the laptop mount. Bottom left: Front view of Irma3D. Two small digital cameras and a 2D laser scanner are attached to the front of the robot for easier navigation. Top middle: The thermal camera on top of the 3D laser scanner that is used to sense thermal properties of the environment. Bottom middle: A DSLR camera is mounted on the 3D laser scanner to provide color information in the point clouds. Top right: The IMU is mounted on the underside of the chassis next to the rear wheel to provide maximum shielding from the magnetic fields that are generated from the motors and the 3D laser scanner. Bottom right: An ExpressCard to RS-232 Adapter is used so that modern laptops without serial ports can interface with the Volksbot motor controller and the IMU device.

12 V 7.2 Ah Panasonic lead-acid batteries. This is two more than in the original design. The chassis has been modified to provide two electric circuits. In addition to extra wiring the modifications include additional elements to the control panel in the back of the Volksbot RT 3. The platform has a variable laptop mount that can fit any reasonably sized laptop. Currently Irma3D operates on a Samsung Q45 Aura laptop with an Intel Core 2 Duo T7100 and $4 \mathrm{~Gb}$ of RAM. The laptop mount has been situated such that the laptop will rest above the control elements of the chassis (see Fig. 2). Therefore, the location of the kill switch has been changed to the rear of the platform to keep it accessible at all times. The physical dimensions of the Volksbot platform are $58 \mathrm{~cm} \times 52 \mathrm{~cm} \times 31.5 \mathrm{~cm}$ with a weight of about $25 \mathrm{~kg}$.

For navigation and obstacle avoidance, the robot is equipped with a SICK LMS 100. This 2D laser scanner is mounted at the front of the chassis and is facing forward, acquiring 2D range scans at a rate of $50 \mathrm{~Hz}$. To fully exploit the $270^{\circ}$ field of view of the SICK LMS 100, the sensor head is positioned slightly above the chassis. The SICK LMS 100 scans with a resolution of $0.5^{\circ}$ and a maximum effective range of about $20 \mathrm{~m}$. To support a human operator when the robot is remote controlled two small webcams of type QuickCam Pro 9000 by Logitech are also attached to the front of the chassis. The motors of the Volksbot are equipped with rotary encoders to measure wheel rotations. This information is used to provide pose estimates of the robot via odometry. The pose estimates are improved using data from the Xsens MTi IMU device that is also attached to the robotic platform. The IMU is susceptible to magnetic interference and must be positioned away from strong magnetic fields to reduce erroneous sensor readings. The motors as well as the laser scanners generate magnetic fields. Therefore, the IMU is fixed to the rear and bottom of the chassis.

The central sensor of Irma3D is the 3D laser scanner VZ-400 by RIEGL Measurement GmbH. The scanner is mounted on top of the volksbot RT 3 chassis. Attached to the top of the scanner is a Canon 1000D DSLR camera. After a 3D scan has been acquired the camera is used to acquire color information for the point cloud. A similar process is done using the Optris PI160 thermal camera which is also mounted on top of the VZ-400 to acquire information about the thermal properties of structures in the point cloud. All cameras on Irma3D are USB devices. With the addition of the gamepad for remotely controlling the robot this adds up to a total of 5 USB plugs that need to be connected. To reduce requirements on the laptop Irma3D is equipped with a USB hub. Both laser scanners transfer information via Ethernet. To enable the laptop to connect to both devices at the same time, Irma3D is also provided with a network switch. The Volksbot motor controller as well as the Xsens MTi communicate via RS232 serial ports. Since modern laptops are rarely equipped with an RS232 port, let alone two, a Delock PCMCIA to RS232 adapter is used to allow for communication. The laptop supplies its own power via the laptop battery. All USB devices draw their power from the laptop. The network switch as well as all other sensors with the exception of the VZ-400 share a power supply with the Volksbot chassis in the form of two of the four lead batteries. The remaining two batteries are dedicated to the VZ-400, as it draws a similar amount of power to the rest of the system.

The VZ-400 is able to freely rotate around its vertical axis to acquire 3D scans even when the robot is not in motion. The fastest it can do this is at $6 \mathrm{~s}$ per rotation. At this speed each point cloud will contain about 750000 points. The minimum angular resolution of a range scan is $0.0024^{\circ}$ in both directions. Given that the scanners vertical field of view is $100^{\circ}$, this equates to more than 6 billion points per scan. The scanner is capable of online Full Wave Transform, may record multiple distance measurements per laser beam and will return not only the range of each echo, but also their amplitude, deviation and a calibrated reflectance value. Amplitude and deviation refer to the parameters of the normal distribution that is fitted into each response. The deviation of a point is a measure for the certainty of the measurement. The amplitude is roughly equivalent to the intensity with which the laser beam was reflected. This value is similar to what other laser scanners return as reflectance, reflectivity or intensity. It should be noted that this is not a good measure of the reflection coefficient of a surface. The intensity of a signal is affected not only by the reflectance properties of the surface but also by the angle of incidence, the distance to the surface, the temperature and other atmospheric conditions. The VZ-400 also returns so-called calibrated relative reflectance values, which attempts to correct for the influence of the distance to the surface.

\subsection{Scan pattern analysis of Irma3D}

Irma3D is a mobile laser scanner that acquires range measurements in a manner decidedly distinct from conventional mobile laser scanners. When Irma3D is in motion, its continually spinning 3D laser scanner will scan the environment in a spiral pattern instead of a regular grid. We will now examine what this 
different mode of mobile laser scanning entails. One advantage has already been mentioned. A mobile laser scanner with a rotating 3D scanner can also be used in stop and go mode to acquire range scans. In fact, this is not a binary either-or decision either. Both modes could be used in combinations with each other. This would help registration, since scans acquired while the vehicle is stopped can be localized more easily. Range measurements taken while in movement could then be used to improve upon the density of the 3D model. The laser scanner is then always in use with no wasted idle time like in static scanning.

Other advantages and disadvantages can be more easily explained when visualizing the scan results that different types of mobile laser scanners acquire in the same environment. For this purpose a simple scanning scenario in a tunnel has been simulated. The $20 \mathrm{~m}$ long tunnel is in the shape of a cylinder with a radius of $4 \mathrm{~m}$ that is cut in half precisely along its axis. Each mobile laser scanner traverses the tunnel along its axis. This scenario as well as the point clouds that result from this simulation are depicted in Fig. 3. It should be noted, that other designs for MLS systems are conceivable as well. For example, a design similar to the stationary 3D scanner by Ohno et al. Ohno et al. (2010) could theoretically be adapted to mobile purposes. In their design a 2D laser scanner is actuated by two servo motors. The two rotational degrees of freedom allow for a variety of more complex scanning patterns. Mobile laser scanning systems that employ rotating 3D laser scanners are easily identifiable by their characteristic spiral pattern. 2D laser scanners in motion always generate regular grid patterns. This is irrespective of how many scanners are used or how they are mounted on the mobile platform. Systems like Irma3D exhibit a more uneven distribution of points.

The point density on a planar surface is only dependant on the distance and angle of that surface in relation to the laser scanner. This holds true for mobile systems with 3D laser scanners as well. In contrast to systems with $2 \mathrm{D}$ scanners though, the angle of a surface to the laser scanner varies with time even when the orientation of the mobile platform does not change. Due to the ever changing orientation of the laser beam with respect to the mobile platform, some time will be spent measuring "ahead" and "behind" of the platform. This has the disadvantage of less measurements falling on the side of the platform. However, details in the environment that can only be measured from certain angles will more likely be picked up by a rotating laser scanner. This issue often arises in urban environments, where many planar surfaces like street signs, fences or even walls may be aligned in an unfortunate manner towards the laser scanner. A street sign that is coplanar with or close to coplanar with the laser plane of a conventional mobile scanner is near invisible. With a rotating $3 \mathrm{D}$ scanner this is not the case.

Another advantage for Irma3D is that the laser beam always "returns" to previously scanned objects in the environment. Note how much the timestamps of measurements near to each other vary for systems like Irma3D in Fig. 3 in contrast to conventional mobile scanners. At both ends of the tunnel this type of mobile laser scanner can acquire range measurements from the other end of the tunnel. This has implications for the registration of the point clouds that will become more obvious in the next sections. To be clear, only measurements taken at different times that are correlated to each other and can be identified to be correlated can be used to compute improved estimates for the position and orientation of the laser scanner at those times. Although all range measurements from a static environment naturally correlate to each other this correlation is not so simple to extract. Point measurements that stem from a single feature in the environment, or from surfaces that are near to each other, are much easier to identify even in noisy data. It is therefore important for registration to use a system that is capable of acquiring such seemingly redundant measurements. A conventional mobile scanner with multiple 2D scanners is capable of this, although to a very limited extent because only a very short period of time elapses between the pass of the first $2 \mathrm{D}$ scanner and the passes of the other 2D scanners. These systems only allow for a trajectory correction in these minuscule time steps.

Finally, there is one more difference in the distribution of range measurements between the two types of mobile laser scanner. The effect is clearly visible when varying the speed of the mobile platform in the simulation. This was done in Fig. 4. The faster the platform, the less time is spent in any given environment. Since the measuring rate remains constant, less points are acquired as a consequence. As with conventional mobile scanners the point density decreases the faster the platform moves. However, as can be seen in the right of Fig. 4 the point distribution to the left and to the right of the mobile laser scanner is not equal. This effect is due to the direction of rotation of the $3 \mathrm{D}$ laser scanner. In the simulation the $3 \mathrm{D}$ scanner was rotating in a clockwise fashion. Thus, the sweeping motion of the scanner on the left side of the platform coincides with the direction of movement of the platform. On the right hand side of the platform both directions are opposed to each other. Consequently, points on the left hand side are more evenly distributed than on the right hand side.

Mathematically, this can be explained as follows. The apparent velocity $v_{l}(t)$ at time $t$ of a laser beam on a wall that is parallel to the direction of motion of the platform and at a distance of $D$ to the laser scanner on its left hand side is given by:

$$
v_{l}(t)=v_{m}+v_{s} D\left(1+\tan ^{2}\left(v_{s} t\right)\right)
$$

assuming that the laser scanner is rotating in a clockwise fashion. Here $v_{m}$ describes the velocity of the platform and $v_{s}$ describes the rotation velocity of the laser scanner. The equivalent apparent velocity $v_{r}(t)$ of the laser beam on the right hand side is given by:

$$
v_{r}(t)=v_{m}-v_{s} D\left(1+\tan ^{2}\left(v_{s} t\right)\right) .
$$

It is clear that $v_{l}(t)$ will always remain positive, whereas $v_{r}(t)$ can be both negative and positive when at least either the forward velocity of the platform is too low, the distance to the surface is too short or the rotation velocity of the scanner is too high. See Fig. 5 for a plot of both functions, with $D$ set to $1 \mathrm{~m}, v_{m}$ to $1 \mathrm{~m} / \mathrm{s}$ and $v_{s}$ set to $\frac{1}{6} \mathrm{~s}$. This represents a challenging indoor scenario for a relatively swiftly moving Irma3D. The interesting range of angles is around $0^{\circ}$, where the laser is perpendicular to the surface. Absolute angles greater than $80^{\circ}$ can be considered unimportant for objects to the side of the mobile laser scanner. The dominating part of both functions is the squared tangent. However, the addition of $v_{m}$ means that the average velocity around $0^{\circ}$ is high for the left side and quite low for the right side. Since the tangent tends towards infinity the average velocity for large absolute angles is similarly high for both sides. The result is that on the right hand side the laser beam quickly approaches a position, remains in that general location for a while and then quickly leaves it again.

While this effect should be kept in mind when choosing the movement speed of Irma3D for specific environments it should be noted that the previous analysis assumes a highly simplified scenario. Furthermore the effect is much less pronounced in reality than what Fig. 4 suggests due to the much higher scan rate of the terrestrial laser scanner. 

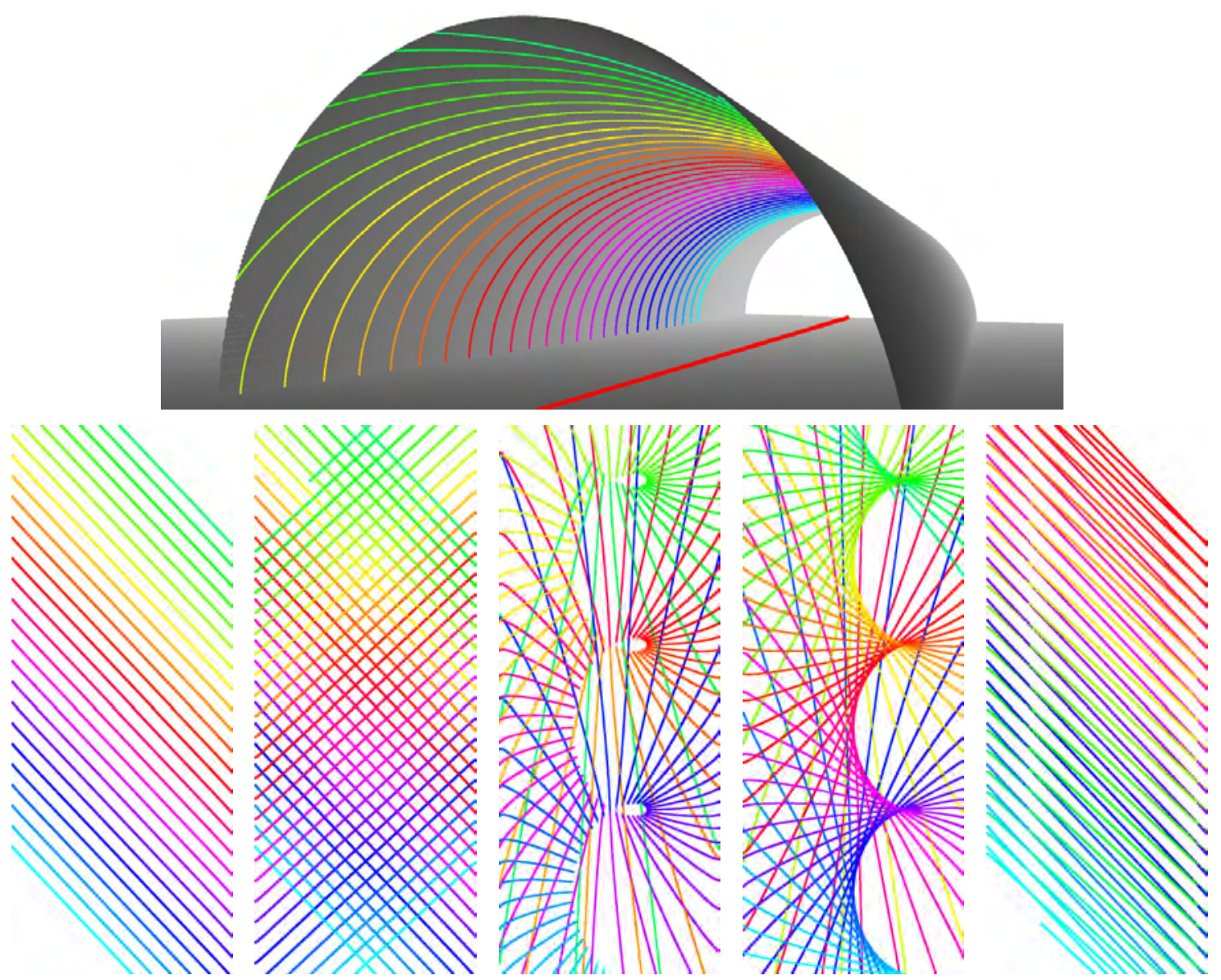

Figure 3: Five different principles of scanning a tunnel were simulated. Top: The tunnel is half a cylinder, truncated with a plane that is collinear to the axis of the cylinder. The mobile laser scanner moves along the axis (red) and acquires range measurements. These are displayed in an orthographic projection as seen from exactly above the tunnel. The points are colored according to the time that they were acquired (from blue to green). The results of five simulated mobile laser scanners from left to right: 1.) A single 2D laser scanner rotated around the yaw axis, such that there is a $45^{\circ}$ angle between the laser plane and the direction of movement. 2.) Two 2D laser scanners rotated around the yaw axis, such that they form a right angle to each other and a $45^{\circ}$ angle between each laser plane and the direction of movement. 3.) A $2 \mathrm{D}$ laser scanner looking sideways, i.e., with a field of view of $-90^{\circ}$ to $90^{\circ}$ is rotating continuously and in a clockwise fashion around the yaw axis of the mobile platform. $0^{\circ}$ is defined as the angle parallel to the floor. 4.) A $2 \mathrm{D}$ laser scanner looking up, i.e., with a field of view of $0^{\circ}$ to $180^{\circ}$ is also rotating in the same fashion. 5.) The same mobile laser scanner as in 1.) except it traverses the tunnel twice. Once from the bottom to the top and then from the top back to the bottom.
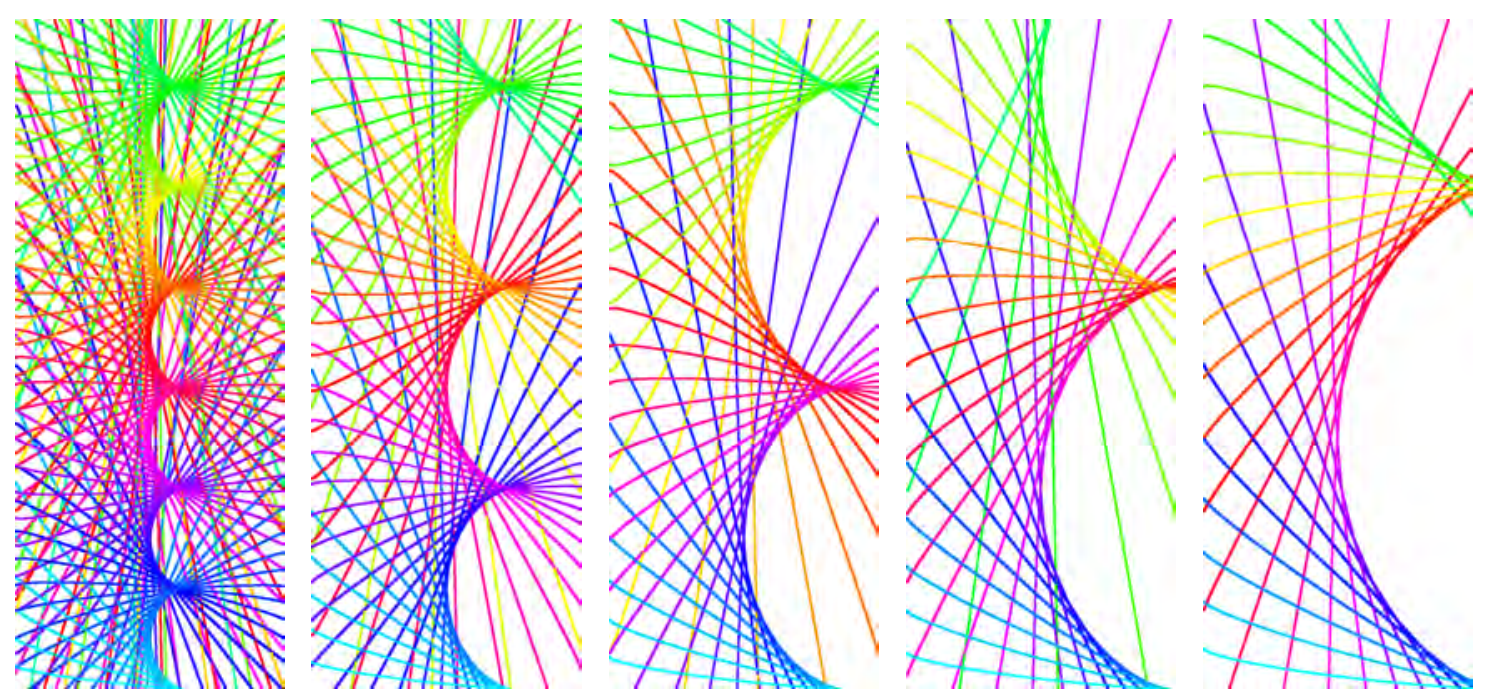

Figure 4: Comparison of the effect of movement speed on the distribution of points for mobile laser scanners of type 4 (see Fig. 3). The scenario is the same cylindrical tunnel as in Fig. 3. The tunnel has a length of $20 \mathrm{~m}$, a radius of $4 \mathrm{~m}$. The 2D scanner requires 6 seconds per rotation and takes $402 \mathrm{D}$ scans per rotation. This last value is unrealistically low and was chosen for the purpose of illustration only. From left to right, the mobile platform moved at a speed of, $1 \mathrm{~m} / \mathrm{s}, 2 \mathrm{~m} / \mathrm{s}, 3 \mathrm{~m} / \mathrm{s}, 4 \mathrm{~m} / \mathrm{s}$ and $5 \mathrm{~m} / \mathrm{s}$. 


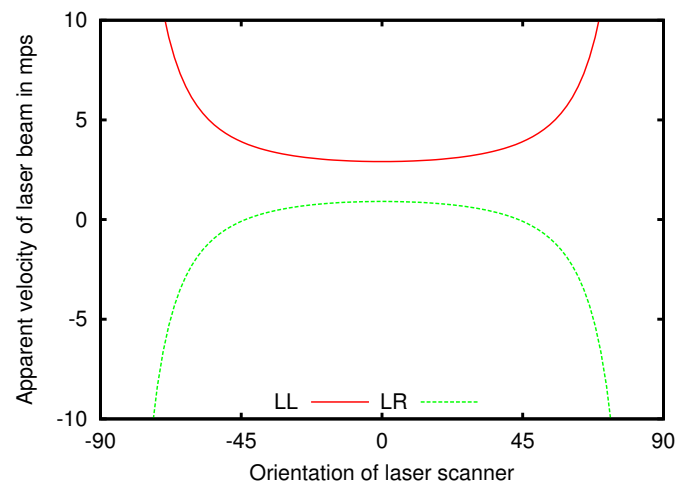

Figure 5: The velocity of the laser beam on surfaces parallel to the motion of the platform and at a distance of $1 \mathrm{~m}$. The platform moves at a speed of $1 \mathrm{~m} / \mathrm{s}$, whereas the laser scanner requires $6 \mathrm{~s}$ for a single rotation in a clockwise fashion

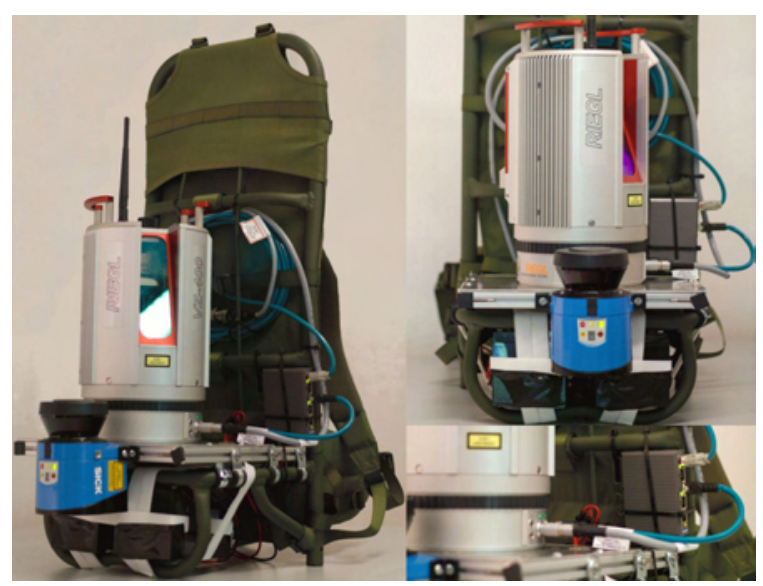

Figure 6: Images of the backpack system. Left: Side view with all of its sensors and equipment. Right: Detailed view of the SICK and the switch.

\subsection{The Backpacking System}

The setup of the backpacking system is strongly influenced by Irma3D. The basis is a Tatonka load carrier where item 24 profiles similar to the volksbot RT 3 chassis have been attached using pipe clamps. Energy is currently provided by two Panasonic $12 \mathrm{~V}$ lead-acid batteries with $12 \mathrm{Ah}$, but to save weight, these will be replaced by lithium polymer battery. Similarly to Irma3D, the backpack features a horizontally scanning SICK LMS 100, which is used to observe the motion of the carrier using a FastSLAM variant. To fully exploit the $270^{\circ}$ field of view of the SICK LMS 100 , the sensor head is positioned slightly above the load carrier. The central sensor of the backpack system is the 3D laser scanner RIEGL VZ-400. The VZ-400 is able to freely rotate around its vertical axis to acquire 3D scans. Due to the setup, however, there is an occlusion of about $100^{\circ}$ due to the backside of the backpack and the human carrier. The backpack is also provided with a network switch to receive the data from the two scanners and to connect the laptop (Samsung Q45 Aura laptop with an Intel Core 2 Duo T7100), which is carried by the human. Please note that the backpack is currently not equipped with an IMU.

Due to the occlusion, it is disadvantageous to constantly spin the VZ-400 as the resulting trajectory has a gap. Currently, our semirigid SLAM solution for optimizing the trajectory (cf. next section) cannot handles these gaps. We have programmed the scanner such that it rotates back-and-forth. Fig. 7 compares the resulting scan patterns.

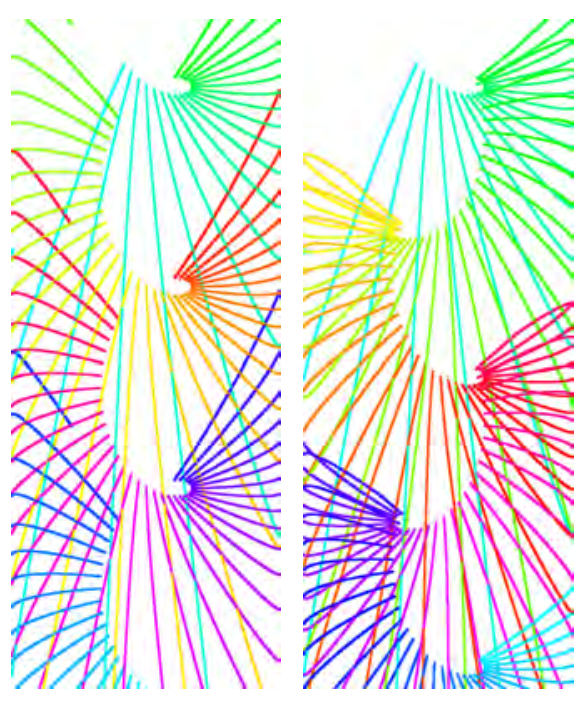

Figure 7: The results of simulated mobile laser scanning missions for the backpack system (cf. Fig. 3). Left: Spinning 3D scanner that is affected by an occlusion of $100^{\circ}$. Right: System that rotates back-and-forth and thus, all laser pulses capture something.

\section{MOBILE MAPPING WITH CONSTANTLY SPINNING SCANNERS}

The following subsections summarize our work in Borrmann et al. (2008); Elseberg et al., 2013 (submitted). These algorithms are suited to turn laser range data acquired with a rotating scanner while the acquisition system is in motion into globally consistent 3D point clouds.

\subsection{High-Precise Registration of Terrestrial 3D Scans}

The basis of our software development is the well-known iterative closest point (ICP) algorithm. Given two 3D point clouds and a rough initial pose estimate, e.g., by the robots odometry, ICP iteratively revises the pose estimation (translation \& rotation with 6 degree of freedom) of the second scan. For doing so, the algorithm selects closest points between the two raw scans and minimizes an error function. Current research in the context of ICP algorithms mainly focuses on fast variants of ICP algorithms.

Pairwise ICP improves the scan pose estimates, but registration errors sum up when adding more scans. Simultaneous Localization And Mapping (SLAM) algorithms use loop closings to bound this error. Recently, we have presented our globally consistent scan matching algorithm, which is a bundle adjustment solution for 3D scans. It extends the ICP algorithm. The input are $\mathrm{n}$ point clouds and its output are improved pose estimates for all scans. In an ICP-like fashion, the algorithm iteratively calculates closest points between all scan pairs as specified in the SLAM graph. Using these point pairs, an improved pose based on least square error minimization is calculated. Please note, that our algorithm does not require any feature extraction.

\subsection{Automatic Calibration for Mobile Mapping}

To acquire high quality range measurement data with a mobile laser scanner system, the position and orientation of every individual sensor must be known. Currently, we are developing algorithmic calibration methods of these systems, i.e., algorithms to establish the parameters that best describe sensor displacements based on the sensor data itself. In this process parameters measured with external instruments are fine-tuned automatically. 


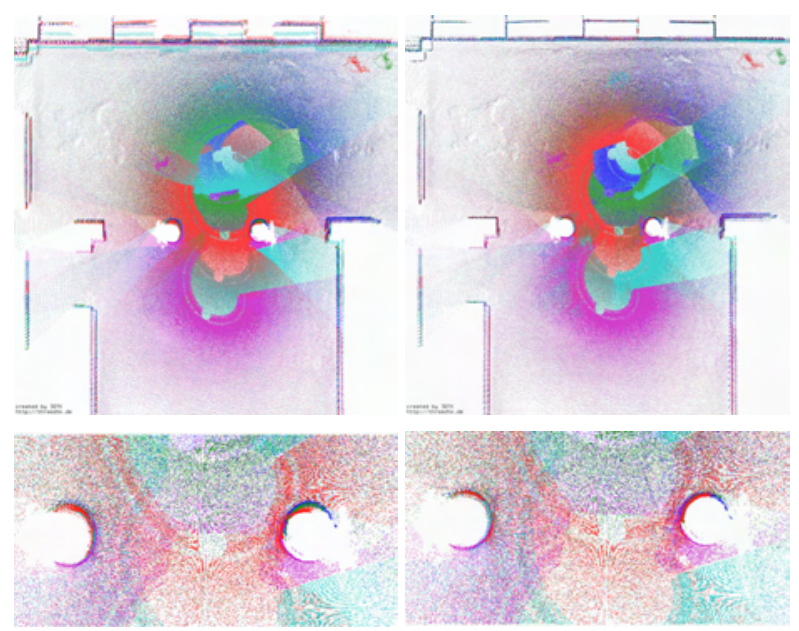

Figure 8: Example of calibrating the robot Irma3D in the field (Ostia Antica, cf. Fig. 1. Left: Before calibration. Right: After calibration.

The state of the art in mobile mapping is: (1) For all sensors determine the position and orientation on the vehicle (calibration), (2) Data acquisition, (3) Extract the trajectory of the vehicle from the sensor data (Kalman-Filter, etc.) (4) georeference the laser measurements with the trajectory to create a $3 \mathrm{D}$ point cloud. The automatic calibration method follows these four steps, but we treat the georeferencing as a function. We have designed an error measure where we determine the quality of the resulting point cloud, based on the 3D points. The entropy is calculated from the closest point correspondences. We can optimize for the position and pose of every sensor, i.e., performing automatic bore sight alignment, and for timing inaccuracies. Aside from sensor misalignment a second source of errors are timing related issues. On our mobile platforms all subsystems need to be synchronized to a common time frame. As our systems are not equipped with a GPS clock or any central trigger mechanism, every sensor uses its own timer and the synchronization is improved by the calibration procedure. Figure 8 shows two results of the calibration. Furthermore, it depicts the typical scan pattern where different revolutions are coded in different colors.

\subsection{Semi-rigid SLAM for Trajectory Optimization}

Besides calibration an even more significant source of errors is the incorrect positioning of the vehicle. Solving this problem requires approaches other than classical rigid SLAM algorithms. An area that provides a solution is the area of non-rigid registration. The approach we have taken optimizes the point cloud using full $6 \mathrm{D}$ poses and is not restricted to a single scanner rotation. Instead we improve scan quality globally in all 6 degrees of freedom for the entire trajectory. In an ICP-like fashion our semirigid SLAM solution computes closest point pairs and includes a timing threshold to prevent matching of consecutive scan slices. Figure 9 presents a resulting point cloud acquired with the backpack system in a long basement room.

\section{RELATED WORK}

Bosse et al. (2012) presented a seminal work about a Hokuyo laser scanner mounted on a spring system. The system is handheld, extremely lightweight and uses a nodding scanner motion to acquire 3D data. Previously, Bosse and Zlot (2009) considered also spinning SICK laser scanners. Their point cloud optimization algorithm considers planar patches extracted from a sweep and deforms the trajectroy using a spline. Also, Stoyanov and

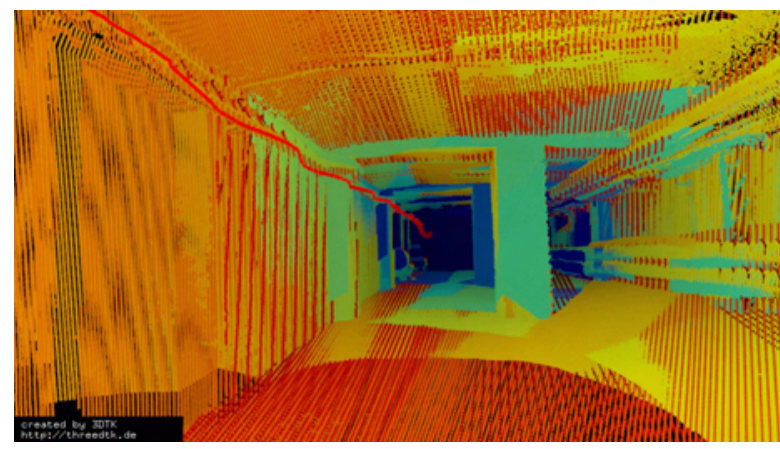

Figure 9: 3D point cloud acquired by the backpack system after applying semi-rigid SLAM. Details are given in Elseberg et al., 2013 (submitted).

Lilienthal (2009) provided a rotating scanner and matched a start and end of a rotation for point cloud optimization. Before that, Wulf et al. (2008) use a rotating SICK scanner for 3D data acquisition. However, different scan patters have not been considered. Holz et al. (2009) built a rotating SICK scanner, where the scanners have been rotated by $45^{\circ}$, similarly to state of the art kinematic scanning systems.

\section{CONCLUSIONS AND FUTURE WORK}

This paper has presented a study of scan patterns for mobile scanning. While many mobile mapping systems use two rigid 2D profilers, an emerging trend is to built systems that scan continuously using a 3D scanner. The resulting scan pattern is affected by the speed of rotation and the velocity of the mobile system, which have to be carefully selected. In the presence of occlusions it is advantageous to use a back-and-forth-scanning pattern.

Future work will concentrate on the backpacking system. Since the system does not use an IMU and only the 2D laser scanner for detecting the horizontal motion, we plan to optimize the resulting point clouds by adding global constraints.

\section{References}

Borrmann, D., Elseberg, J., Lingemann, K., Nüchter, A. and Hertzberg, J., 2008. Globally consistent 3 d mapping with scan matching. Journal Robotics and Autonomous Systems (JRAS) 56(2), pp. 130-142.

Bosse, M. and Zlot, R., 2009. Continuous 3D Scan-Matching with a Spinning 2D Laser. In: Proceedings of the IEEE International Conference on Robotics and Automation (ICRA '09), pp. 4312-4319.

Bosse, M., Zlot, R. and Flick, P., 2012. Zebedee: Design of a springmounted 3-d range sensor with application to mobile mapping. IEEE Transactions on Robotics (TRO) 28(5), pp. 1104-1119.

Elseberg, J., Borrmann, D. and Nüchter, A., 2013 (submitted). Algorithmic solutions for computing accurate maximum likelihood 3D point clouds from mobile laser scanning platforms.

Holz, D., Droeschel, D., Behnke, S., May, S. and Surmann, H., 2009. Mobile Robots Navigation. InTech, chapter Fast 3D Perception for Collision Avoidance and SLAM in Domestic Environments.

Ohno, K., Tadokoro, S., Nagatani, K., Koyanagi, E. and Yoshida, T., 2010. Trials of 3-D Map Construction using the Tele-operated tracked Vehicle Kenaf at Disaster City. In: Proceedings ICRA, pp. 2864-2870.

Stoyanov, T. and Lilienthal, A. J., 2009. Maximum Likelihood Point Cloud Acquisition from a Mobile Platform. In: Proceedings of the IEEE International Conference on Advanced Robotics (ICAR '09), pp. $1-6$.

Wulf, O., Nüchter, A., Hertzberg, J. and Wagner, B., 2008. Benchmarking Urban 6D SLAM. Journal of Field Robotics (JFR) 25(3), pp. 148-163. 